\title{
Procoagulant and anticoagulant factor abnormalities following the Fontan procedure: Increased factor VIII may predispose to thrombosis
}

\author{
Kirsten C. Odegard, $\mathrm{MD}^{\mathrm{a}}$ \\ Francis X. McGowan, Jr, MD \\ David Zurakowski, $\mathrm{PhD}^{\mathrm{c}}$ \\ James A. DiNardo, MDa \\ Robert A. Castro, $\mathrm{MT}^{\mathrm{a}}$ \\ Pedro J. del Nido, MD \\ Peter C. Laussen, MBBS ${ }^{\mathrm{a}}$
}

From the Departments of Anesthesia, Cardiac Surgery, and Biostatistics, Children's Hospital, Boston, and Harvard Medical School, Boston, Mass.

Received for publication April 25, 2002; revisions received July 17, 2002; accepted for publication Aug 27, 2002

Address for reprints: Kirsten C. Odegard, MD, Cardiac Anesthesia Service, 300 Longwood Avenue, Children's Hospital, Boston, MA 02115 (E-mail: kirsten.odegard@tch. harvard.edu).

J Thorac Cardiovasc Surg 2003;125:1260-7

Copyright $\odot 2003$ by The American Association for Thoracic Surgery

$0022-5223 / 2003 \$ 30.00+0$

doi:10.1016/S0022-5223(02)73605-2

Objective: Using age-matched controls, this study prospectively evaluated coagulation factor abnormalities and hemodynamic variables in children who had undergone the Fontan operation.

Methods: Coagulation factors were assayed in 20 children (mean age $6.4 \pm 2.9$ years), at a mean $3.7 \pm 2.3$ years after the Fontan procedure; 24 healthy children (mean age $6.8 \pm 2.8$ years) were assayed as controls. Concentration of factors II, $\mathrm{V}$, VII, VIII, IX, X; ATIII; plasminogen; proteins C and S; fibrinogen; serum albumin; and liver enzymes were measured. Normal reference intervals based on the control patients were determined using 95\% confidence limits. Patient demographic, hemodynamic variables, and elapsed time after the Fontan procedure were evaluated as possible predictors of coagulation abnormalities.

Results: Concentrations of protein C; factors II, V, VII, X; plasminogen; and ATIII were significantly lower in Fontan patients compared with age-matched controls $(P$ $<.01)$; factor VIII was significantly elevated in 6 patients $(35 \%), 2$ of whom had a thromboembolic event. A higher superior vena cava pressure was predictive of an elevated factor VIII level $(P=.003)$. No other specific hemodynamic variables were predictive of a procoagulant or anticoagulant abnormality.

Conclusion: Procoagulant and anticoagulant factor levels were significantly lower in patients after the Fontan operation independent of hemodynamic variables peculiar to the Fontan circulation. Increased factor VIII level requires further evaluation as a cause of thrombosis in patients with Fontan physiology and may also indicate a subset of these patients in whom anticoagulation is indicated.

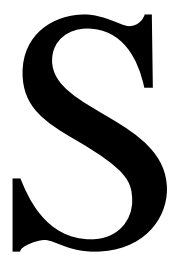

ince the original Fontan operation was described, several modifications have been undertaken that have improved early and late morbidity and mortality. Survival following the fenestrated lateral tunnel Fontan procedure of $93 \%$ at 5 years and $91 \%$ at 10 years has recently been reported. ${ }^{1}$ Nevertheless, complications including ventricular dysfunction, thromboembolic events, dysrythmias, and protein losing enteropathy may compromise longer-term function and outcome. ${ }^{2}$

The incidence of thromboembolic events in patients with Fontan physiology is uncertain but has been reported to be as high as $20 \%$ to $33 \%$. $^{3-6}$ Abnormal coagulation parameters following the Fontan procedure involving both pro- and anticoagulant factors have been described. ${ }^{7-10}$ These studies suggested that the alterations in the coagulation factors found in some Fontan patients are a direct 
consequence of the anatomic and physiologic changes that result from the Fontan operation. The nature of the "Fontan" circulation with elevated central venous pressure, low flow with possible stasis through the atrial baffle and pulmonary circulation, atrial dysrhythmias, hepatic dysfunction, and increased resting venous tone are some of the suggested factors. However, we recently reported that pro- and anticoagulant factor abnormalities occur earlier in the course of staged surgical palliation for single ventricle disease. ${ }^{11,12}$ Whether this is due to other physiologic derangements such as cyanosis, low cardiac output, venous congestion, or perhaps a genetic predisposition is unknown.

Previous studies have evaluated only a small number of coagulation factor abnormalities after the Fontan operation, have not used age-matched controls, and have not undertaken a detailed analysis of hemodynamic variables that could alter coagulation factor levels. In this single-center, prospective, cohort study, coagulation factor abnormalities after the Fontan operation were evaluated, along with hemodynamic variables peculiar to the Fontan circulation that could contribute to coagulation abnormalities.

\section{Materials and Methods}

After obtaining institutional review board approval and informed parental consent, 20 children who had undergone a prior Fontan operation were enrolled in this prospective study. Patients were excluded if they had preexisting hematological disorders, concurrent coagulopathies, or if they were anticoagulated with coumadin; $16(80 \%)$ patients had been receiving aspirin but this was discontinued 7 to 10 days prior to blood draw.

All patients underwent general anesthesia; 13 patients for cardiac catheterization, 5 patients for permanent pacemaker placement/revision, 1 patient for aortic valvuloplasty, and 1 patient for elective noncardiac surgery. Blood samples from all patients ( 8 $\mathrm{mL}$ ) were obtained from a venous catheter or an indwelling arterial catheter after induction of general anesthesia. Measured parameters included hemoglobin ( $\mathrm{Hgb})$, hematocrit (Hct), platelet count, prothrombin time (PT), and activated partial prothrombin time (APTT). Inhibitors of coagulation measured included protein $\mathrm{C}$, protein $\mathrm{S}$, plasminogen, and antithrombin III; the procoagulant factors measured were II, V, VII, VIII, IX, X, and fibrinogen.

The Coulter T 660 (Beckman Coulter, Inc, Miami, Fla, USA) automated hematology analyzer was used to measure $\mathrm{Hgb}$, Hct, and platelets; coefficients of variation (\%) within and between assays 3 different Hgb levels were: 1.8, 1.0, 0.9; for Hct: 1.8, 1.2, 1.5; and for platelets: $4.8,2.7,1.8$, respectively.

The ACL 3000 plus (Automated Coagulation Laboratory, Beckman Coulter Inc, Miami, Fla) was used to assay individual procoagulant and anticoagulant factors as well as the PT and APTT. The ACL contains two measuring systems: (1) nephelometry, which is used to detect clot formation as the endpoint, and (2) photometry, which is used for chromogenic substrate assays. Blood for these assays was collected in citrated plasma $(3.2 \%$ buffered sodium citrate) from an indwelling cannula from which $10 \mathrm{~mL}$ of blood had been aspirated to remove residual heparin. The blood was immediately centrifuged at $13,000 \mathrm{rpm}$ for 5 minutes and the plasma layer removed. The PT, APTT, and fibrinogen were measured immediately using nephelometry (coefficients of variation [\%] respectively: PT: 1.2, 2.3; APTT: 2.1, 4.8; fibrinogen: 3.1, 5.5). Remaining plasma was stored at $-70^{\circ} \mathrm{C}$ in $200 \mu \mathrm{L}$ aliquots for batch performance of the other coagulation assays, as described below.

Protein $\mathrm{C}$ and $\mathrm{S}$ activity were both measured using functional clotting assays. Protein $\mathrm{C}$ activity was determined based on the prolongation of the APTT using the Staclot Protein C kit (Diagnostica Stago, Asnieres-Sur-Seine, France) according to the manufacturer's directions. In this assay, activated protein $\mathrm{C}$ inhibits factor $\mathrm{V}$ and VIII activity, thus prolonging the APTT of a system in which all the factors with the exception of protein $\mathrm{C}$ are present in excess; protein $\mathrm{C}$ is derived from the sample being tested (coefficients of variation [\%]: 1.8 and 2.4).

Protein $\mathrm{S}$ activity was determined based on the principle of factor Va inhibition using the Staclot Protein S kit (Diagnostica Stago, Asnieres-Sur-Seine, France). The principle of the test is based upon the cofactor activity of protein $S$, which enhances the anticoagulant action of activated protein $\mathrm{C}$. This enhancement is reflected by the prolongation of the clotting time of a system enriched with factor $\mathrm{Va}$ (coefficients of variation [\%]: 7.9 and 3.8).

Extrinsic coagulation factors (factors II, V, VII, and X) were each determined by performing a modified prothrombin time assay. Intrinsic coagulation factors (factors VIII and IX) were determined by performing a modified activated partial thromboplastin time. In these assays, correction of the clotting time of plasma specifically deficient in the factor being tested is proportional to the concentration (activity \%) of that factor in the patient plasma, interpolated from a calibration curve (control plasma deficient in factors II, V, VII, VIII, IX, or X was obtained from Instrument Laboratory, Lexington, Mass; coefficient of variation [\%] respectively: II: 2.3, 2.7; V: 3.3, 2.3; VII: 2.5, 2.2; VIII: 4.9, 3.7; IX: 3.2, $3.1 ; \mathrm{X}: 3.0,2.0)$.

Antithrombin III activity was determined using a synthetic chromogenic substrate assay that is based upon factor Xa inactivation (Antithrombin III, Instrument Laboratory, Lexington, Mass, USA; coefficients of variation [\%]: 4.3 and 2.8). Plasma plasminogen was activated through reaction with an excess of streptokinase in the presence of fibrinogen. Plasminogen content was then determined based on a synthetic chromogenic substrate assay according to the manufacturer's directions (Plasminogen, Instrument Laboratory, Lexington, Mass; coefficients of variation [\%]: 4.5 and 3.7).

Because altered hepatic dysfunction can contribute to coagulation factor abnormalities, serum alkaline phosphatase, gammaglutamyl transferase, alanine transaminase, aspartate transaminase, total bilirubin, albumin, and total protein were measured in all patients and compared with normal values for our laboratory.

\section{Age-Matched Control Coagulation Parameters}

Coagulation factor levels vary with age and maturation of the coagulation system, as well as with the reagents used to perform the different tests. To adjust for this, after informed written parental consent was obtained, 24 healthy children ( 9 females, 15 males), mean age $6.8 \pm 2.8$ years (range 3.0 to 13.5 years), undergoing minor day surgery procedures were also studied. Blood was taken from each patient after induction of anesthesia and 


\section{TABLE 1. Cardiac diagnoses of patients after the Fontan procedure}

\begin{tabular}{lll}
\hline Diagnoses & $\mathbf{n}$ & \multicolumn{1}{c}{ Prior surgery } \\
\hline $\begin{array}{l}\text { Morphologic LV } \\
\text { DILV/MA }\end{array}$ & 1 & BTS, BDG, FenFon \\
DILV & 1 & PAB, BDG, FenFon \\
DILV/TGA & 3 & 1 St1, FenFon, PPM/1 BDG, PPM, FenFon/1 \\
& \multicolumn{2}{c}{ BDG, Ex.FenFon } \\
TA/PS & 1 & BTS, FenFon \\
TGA/PA & 1 & BTS, FenFon \\
Morphologic RV & & \\
DORV/VSD & 2 & 1 PAB, BDG, FenFon/1 PAB, FenFon \\
DORV/MA & 1 & PAB, BDG, FenFon \\
DORV/PS/MS & 1 & BDG, FenFon \\
TA/PS & 1 & BTS, FenFon \\
TGA/PA & 1 & BTS, FenFon \\
HLHS & 8 & 8 St1, BDG/7, FenFon/1 Ex. FenFon/1 PPM \\
Heterotaxy & 1 & BTS, BDG, FenFon
\end{tabular}

$\overline{B D G}$, Bidirect Glenn procedure; $B T S$, Blalock-Taussig shunt; DILV, double inlet left ventricle; DORV, double outlet right ventricle; FenFon, Fenestrated Fontan procedure; Ex.FenFon, extracardiac fenestrated Fontan procedure; $H L H S$, hypoplastic left heart syndrome; $M A$, mitral stenosis; $M S$, mitral stenosis; $P A$, pulmonary atresia; $P A B$, pulmonary artery band; $P P M$, permanent pacemaker; $P S$, pulmonary stenosis; $S t 1$, stage 1 procedure (Norwood); $T A$, tricuspid atresia; TGA, transposition of great arteries; VSD, ventricular septal defect.

placement of an intravenous catheter and collected into citrated plasma tubes (3.2\% buffered sodium citrate). Blood samples were immediately centrifuged at $13,000 \mathrm{rpm}$ for 5 minutes and the plasma stored at $-70^{\circ} \mathrm{C}$ for subsequent batch analyses. Proteins $\mathrm{C}$ and S, plasminogen, fibrinogen, ATIII, and factors II, V, VII, VIII, IX, $\mathrm{X}$ were analyzed as described above.

\section{Potential Hemodynamic Factors}

Ventricular morphology (morphologic right or left systemic ventricle), ventricular function, and atrioventricular valve and semilunar valve function were assessed by preoperative echocardiography. Superior vena cava $\mathrm{O}_{2}$ saturation $\left(\mathrm{SvO}_{2}\right)$, the ratio of pulmonary to systemic blood flow (Qp/Qs), superior vena cava pressure (SVCp), pulmonary artery pressure (PAP), pulmonary vascular resistance (PVR), and systemic ventricular end-diastolic pressure (EDP) were all assessed by cardiac catheterization, either the day of data collection or within 3 months prior to the procedure. The length of time following the Fontan operation was also examined as a potential variable contributing to coagulation abnormalities.

\section{Statistical Analysis}

Normal ranges for each of the 11 coagulation variables and proteins were determined using the empirical $95 \%$ confidence intervals. Controls and Fontan patients were compared using the 2-sample Student $t$ test after testing for normality (Kolmogorov-Smirnov test). The sample sizes of 24 controls and 20 Fontan patients provided $85 \%$ power (at an $\alpha$ level of 0.05 , effect size $=1$ ) to detect a significant difference between the groups for each of the coagulation factors (version 4.0, nQuery Advisor, Statistical So- lutions, Boston, Mass). Univariate and multivariate logistic regression using maximum likelihood estimation was performed to identify potential predictors of a coagulation abnormality. Variables evaluated included age, weight, gender, ventricle morphology (left or right), EDP, $\mathrm{SvO}_{2}, \mathrm{SVCp}$, Qp/Qs ratio, PAP, PVR, ventricular function, atrioventricular valve regurgitation, and time since Fontan operation. Mean coagulation levels in patients with and without fenestration closure were assessed using Student $t$ tests. Multiple linear regression was used to develop a prediction equation for coagulation levels with adjusted $\mathrm{R}^{2}$ as the measure of goodnessof-fit. Statistical analysis was conducted using the SPSS software package (version 11.0, SPSS Inc, Chicago, Ill). Values are given as mean \pm SD unless otherwise stated. Significance was set at $P<$ 0.05 for 2-tailed comparisons.

\section{Results}

The mean age for the 20 patients ( 3 girls and 17 boys) was $6.4 \pm 2.9$ years (range 2.6 to 14.5 years) and mean weight was $19.8 \pm 9.8 \mathrm{~kg}$. Patient diagnoses and prior procedures are summarized in Table 1. Eighteen patients had undergone a fenestrated intra-atrial lateral tunnel Fontan operation. Two patients had undergone an extracardiac Fontan operation, one of which was fenestrated at the time of surgery and the other fenestrated in the catheterization laboratory because of protein losing enteropathy (PLE). Three patients (15\%) had signs of PLE with hypoproteinemia and ascites; 2 of these patients had elevated SVCp (20 and $20 \mathrm{~mm} \mathrm{Hg}$ ) and EDP (12 and $14 \mathrm{~mm} \mathrm{Hg}$ ), respectively. The follow-up period between the Fontan operation and inclusion in our study was $3.7 \pm 2.3$ years (range 8.5 months to 9.5 years).

Thirteen patients had a morphologic right ventricle and 7 had a morphologic left ventricle. There were no differences in measured hemodynamics based on ventricular morphology. Hemodynamic variables as assessed either by echocardiography or cardiac catheterization are shown in Table 2.

\section{Normal Ranges}

For the 24 control subjects ( 9 girls, 15 boys), the mean age was $6.8 \pm 2.8$ years (range 3.5 to 13.5 years). The normal reference ranges for coagulation factors are shown in Table 3. Normal ranges were based on the empirical $95 \%$ confidence intervals derived from the 2.5 and 97.5 percentile confidence limits.

\section{Coagulation Abnormalities}

The comparison between coagulation factors in Fontan patients and healthy controls is summarized in Table 4. Significant reductions in the levels of both procoagulant factors and inhibitors of coagulation were detected in all 20 Fontan patients. Concentration of protein C; factors II, V, VII, X; plasminogen; and ATIII were significant lower in Fontan patients compared with age-matched controls $(P<.01)$. Using univariate and multivariate logistic regression, no significant relationship between these coagulation abnormalities and gender, weight, prior procedure, serum albu- 
TABLE 2. Hemodynamic characteristics in Fontan patients

\begin{tabular}{lc}
\hline Variable & $\mathbf{n}(\%)$ \\
\hline Ventricular function & \\
Normal & $13(69 \%)$ \\
Mildly depressed & $5(26 \%)$ \\
Moderately severely depressed & $1(5 \%)$ \\
AVVR & \\
No regurgitation & $4(21 \%)$ \\
Trivial & $3(16 \%)$ \\
Mild-moderate & $12(63 \%)$ \\
EDP (mean mm Hg) & $8.2 \pm 3.7$ \\
SVCp (mean mm Hg) & $12.9 \pm 4.3$ \\
TPG (mean mm Hg) & $5.4 \pm 1.7$ \\
PVR (Wood units) & $1.9 \pm 0.6$ \\
Qp/Os & $0.85 \pm 0.2$ \\
$\mathrm{PaO}_{2}$ mm Hg & $59.5 \pm 8.2$ \\
$\mathrm{SvO}_{2}(\%)$ & $66 \pm 0.1$ \\
$\mathrm{SpO}_{2}(\%)$ & $88 \pm 0.1$ \\
\hline
\end{tabular}

Data expressed as number (\%), and mean \pm standard deviation. AVVR, Atrioventricular valve regurgitation; $C V P$, superior vena cava pressure; $E D P$, end-diastolic pressure; $P A P$, pulmonary artery pressure; $P O_{2}$, oxygen tension; $P V R$, pulmonary vascular resistance; $Q p / Q s$, pulmonary to systemic blood flow; $\mathrm{SpO}_{2}$, oxygen saturation; $\mathrm{SvO}_{2}$, superior vena cava $\mathrm{O}_{2}$ saturation; $T P G$, transpulmonary gradient.

min, the hemodynamic variables previously listed (diagnosis, right or left ventricle morphology, ventricular function, AVVR, PVR, oxygen saturation, $\mathrm{SVO}_{2}, \mathrm{Qp} / \mathrm{Qr}$ ratio, $\mathrm{PO}_{2}$, SVCp, transpulmonary gradient, and EDP) or the time period after the Fontan procedure could be identified (all $P>$ $.20)$.

In contrast, factor VIII was significantly elevated in Fontan patients compared with controls $(P<.04$; Figure 1$)$. Six patients $(35 \%)$ had markedly elevated factor VIII levels $(>150 \%$ activity) compared with the controls. Multiple linear regression analysis indicated that SVCp was a strong predictor of factor VIII levels and this relationship was maintained after controlling for all other hemodynamic and laboratory variables (adjusted $\mathrm{R}^{2}=0.74, P=.003$ ). The relationship between SVCp and factor VIII is illustrated in Figure 2. The predictive equation derived from this model was: Factor VIII $(\%)=12.7 \times \mathrm{SVCp}-28$.

No significant correlations were detected between time since the Fontan operation and any of the 11 coagulation factors or proteins (all $P>.2$ ).

\section{Hemostatic Variables and Liver Function}

The mean Hct, platelet count, PT, APTT, and liver function tests (LFTs) for Fontan patients are shown in Table 5. Partial thromboplastin time (PTT) was abnormally elevated in $29 \%$ of the patients. In all but three patients, abnormal PT and/or PTT values corrected when calibration plasma (Instrumentation Laboratory Company, Lexington, Mass) was added in a 50:50 ratio to patient's plasma. Calibration plasma is obtained from healthy donors and is processed to
TABLE 3. Normal ranges for coagulation factors and protein levels

\begin{tabular}{lrrc}
\hline Variable & Median & Mean \pm SD & Normal range* $^{*}$ \\
\hline Fibrinogen (mg/L) & 321 & $315 \pm 107$ & $104-570$ \\
Factor II (\%) & 92 & $97 \pm 17$ & $71-130$ \\
Factor V (\%) & 107 & $106 \pm 23$ & $76-160$ \\
Factor VII (\%) & 92 & $97 \pm 24$ & $67-172$ \\
Factor VIII (\%) & 99 & $98 \pm 25$ & $43-143$ \\
Factor IX (\%) & 78 & $81 \pm 22$ & $52-136$ \\
Factor X (\%) & 91 & $94 \pm 17$ & $66-126$ \\
Antithrombin III (\%) & 109 & $107 \pm 16$ & $50-127$ \\
Plasminogen (\%) & 102 & $100 \pm 14$ & $77-134$ \\
Protein C (\%) & 97 & $98 \pm 23$ & $56-147$ \\
Protein S (\%) & 83 & $87 \pm 17$ & $69-133$ \\
\hline
\end{tabular}

*Normal ranges were based on the empirical $95 \%$ confidence intervals derived from the 2.5 and 97.5 percentile limits $(n=24)$, except fibrinogen ( $\mathrm{n}=21) . S D$, Standard deviation.

maintain the characteristics of normal plasma. Normalization by calibration plasma indicates one or more factor deficiencies as the cause of the PT or PTT prolongation (as opposed to circulating anticoagulants such as heparin). Three patients did not normalize with calibration plasma for PTT, which was interpreted as heparin contamination; these patients were excluded from PTT evaluation. Three patients failed to normalize with calibration plasma; because protein $\mathrm{C}$ and factor VIII and IX assays are also based upon prolongation of the PTT, their protein C and factor VIII and factor IX data were excluded from analysis.

Liver function tests are also shown in Table 5. Several patients had small elevations of aspartate aminotransferase and alanine aminotransferase, but mean values were within the normal range; gammaglutamyl transpeptidase was elevated in 9 patients $(45 \%)$, and 5 patients $(25 \%)$ had signs of hypoproteinemia (albumin $<3 \mathrm{~g} / \mathrm{dL}$ ), including the 3 patients who carried the clinical diagnosis of PLE. There were no correlations between abnormal liver function tests, hemodynamic variables such as elevated SVCp, or coagulation factor abnormalities.

\section{Thromboembolic Events}

Two patients in our series had a history of significant thromboembolic events. Factor VIII levels for these 2 patients were significantly higher than the other 15 patients $(239 \pm 30 \%$ versus $116 \pm 58 \%$, respectively, $P=.01$, Student $t$ test). One 5-year-old male with PLE was admitted with severe ventricular dysfunction 2.5 years after his Fontan operation. Thrombus in his common atrium and superior vena cava was detected. He was ultimately placed on extracorporeal membrane oxygenation for low cardiac output state and underwent successful orthotopic cardiac transplant. This patient's coagulation profile measured 5 months prior to the event mentioned above was significant for a low normal protein $\mathrm{S}$ level, elevated factor $\mathrm{V}$ and IX levels 


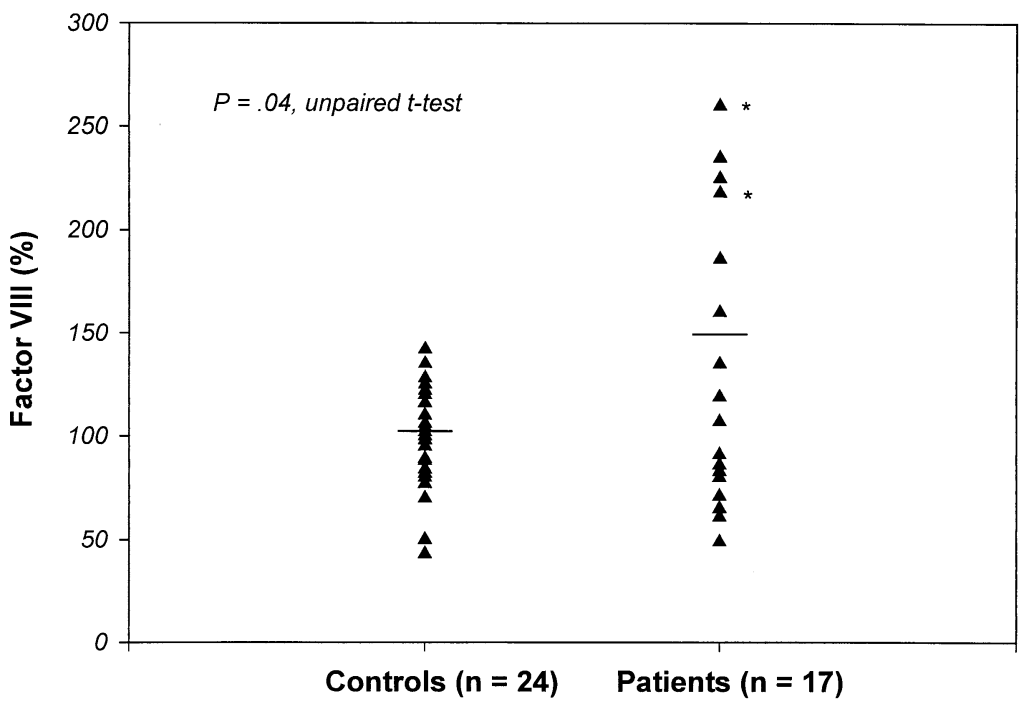

Figure 1. Factor VIII activity in Fontan patients and age-matched controls. Mean factor VIII levels were significantly higher in patients than controls $\left(131 \pm 68 \%\right.$ versus $98 \pm 25 \%, P=.04$. Asterisks $\left({ }^{*}\right)$ indicate the 2 patients who clotted. The mean factor VIII level for each group is marked by a small horizontal bar.

TABLE 4. Coagulation variables for controls and post-Fontan patients

\begin{tabular}{|c|c|c|c|c|c|}
\hline Variable & $\begin{array}{l}\text { Controls } \\
(n=24)\end{array}$ & $\begin{array}{l}\text { Post-Fontan } \\
(\mathrm{n}=20)\end{array}$ & $P$ value ${ }^{*}$ & $\begin{array}{l}\text { No. }(\%) \text { below } \\
\text { normal range }\end{array}$ & $\begin{array}{c}\text { No. }(\%) \text { above } \\
\text { normal range }\end{array}$ \\
\hline Fibrinogen (mg/L) & $315 \pm 107$ & $254 \pm 74$ & $<.05$ & $0(0)$ & $0(0)$ \\
\hline Factor II (\%) & $97 \pm 17$ & $72 \pm 10$ & $<.01$ & $8(40)$ & $0(0)$ \\
\hline Factor $\mathrm{V}(\%)$ & $106 \pm 23$ & $72 \pm 28$ & $<.01$ & $10(50)$ & $0(0)$ \\
\hline Factor VII $(\%)$ & $97 \pm 24$ & $53 \pm 19$ & $<.01$ & $17(85)$ & $0(0)$ \\
\hline Factor VIII (\%) & $98 \pm 25$ & $131 \pm 68$ & .04 & $0(0)$ & $6(35)$ \\
\hline Factor IX (\%) & $81 \pm 21$ & $72 \pm 31$ & .28 & $4(24)$ & $0(0)$ \\
\hline Factor $\mathrm{X}(\%)$ & $94 \pm 17$ & $73 \pm 15$ & $<.01$ & $5(25)$ & $0(0)$ \\
\hline Antithrombin III (\%) & $107 \pm 16$ & $84 \pm 24$ & $<.01$ & $2(10)$ & $1(5)$ \\
\hline Plasminogen $(\%)$ & $100 \pm 14$ & $83 \pm 18$ & $<.01$ & $6(30)$ & $0(0)$ \\
\hline Protein C (\%) & $98 \pm 22$ & $66 \pm 15$ & $<.01$ & $3(18)$ & $0(0)$ \\
\hline Protein S (\%) & $87 \pm 17$ & $80 \pm 14$ & .17 & $4(22)$ & $0(0)$ \\
\hline
\end{tabular}

${ }^{*} P$ values were determined by the 2-sample Student $t$ test. All variables are expressed in terms of the mean $\pm S D$. $n=24$ for controls, except for fibrinogen $(n=21) ; n=20$ for Fontan patients except for factor VIII, factor IX, and protein $C(n=17)$ and for protein $S(n=18)$.

(138\% and $132 \%$, respectively), and a markedly elevated factor VIII level of $260 \%$. LFTs were within normal levels except an albumin level of $1.2 \mathrm{~g} / \mathrm{dL}$.

The second patient was a 8 -year-old boy who had previously undergone an extracardiac nonfenestrated Fontan procedure. He developed progressive PLE and ascites and was admitted for creation of a fenestration in the Fontan baffle. Within hours of the procedure the fenestration became obstructed by thrombus and he was treated with fibrinolytic agents and heparin. He was subsequently discharged home on coumadin. This patient's coagulation profile on the morning of the procedure (prior to thrombus formation) revealed a normal PT, APTT, and protein C and S levels but markedly elevated factor VIII level (218\%) and elevated factor IX (125\%). LFTs were within normal levels with an albumin of $2.1 \mathrm{~g} / \mathrm{dL}$.

No other patients demonstrated clinical evidence of thromboembolic events, and no intracardiac thrombus was detected by transthoracic echocardiography. The majority of the patients (16/20) were discharged home on aspirin alone; 1 patient was discharged on Coumadin; and 3 patients were not given any antiplatelet or anticoagulant therapy.

\section{Discussion}

In this prospective study of coagulation profiles in children with complex single ventricle congenital heart disease who had undergone a prior Fontan operation, abnormalities of 


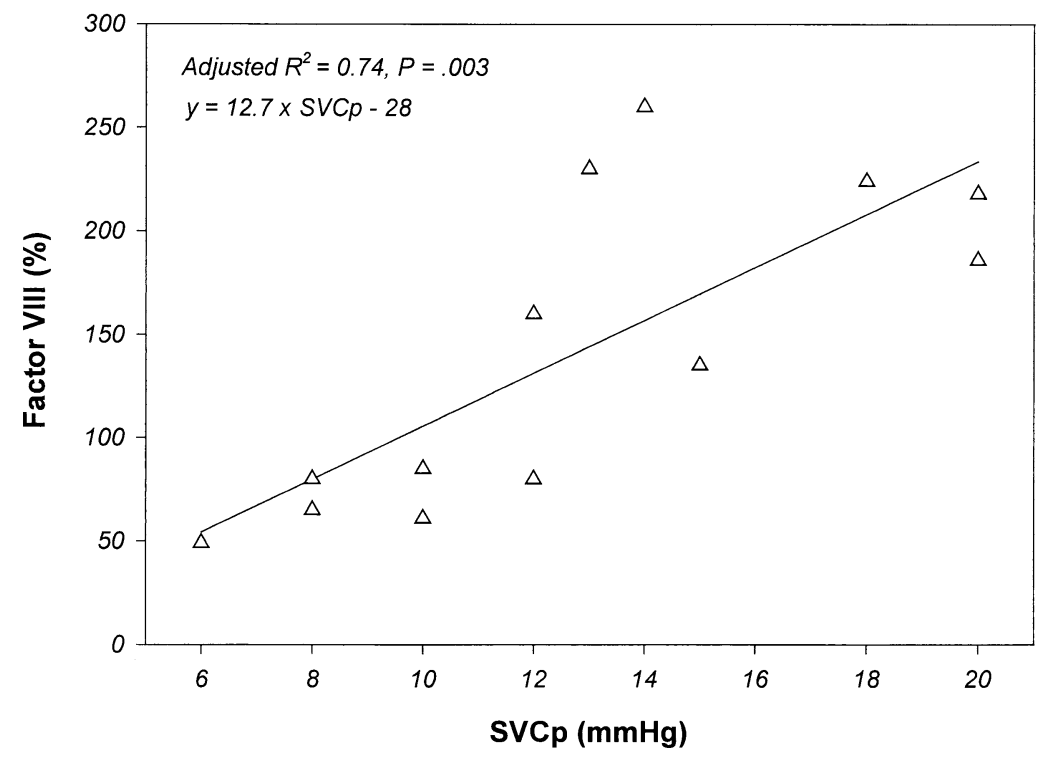

Figure 2. Scatter diagram of the empirical data showing the positive correlation between factor VIII activity as a function of SVC pressure in Fontan patients. The fitted model showed a good linear fit $\left(R^{2}=0.74\right)$ for the regression equation: $y=12.7 x-28$ (solid line).

TABLE 5. Laboratory values for Fontan patients

\begin{tabular}{lcccc}
\hline Variable & $\mathbf{n}$ & Mean \pm SD & Normal range & $\begin{array}{c}\text { Number (\%) above or } \\
\text { below normal range }\end{array}$ \\
\hline Hematocrit (\%) & 19 & $39 \pm 5$ & $33-55$ & $2(11)$ \\
Platelet count $\left(\times 10^{3}\right)$ & 19 & $221 \pm 59$ & $130-400$ & $1(5)$ \\
PT (seconds) & 19 & $12.9 \pm 1.4$ & $11-13$ & $6(32)$ \\
PTT (seconds) & 17 & $38.6 \pm 22$ & $27-37$ & $5(29)$ \\
Albumin (g/dL) & 20 & $3.2 \pm 0.7$ & $3.0-4.6$ & $5(25)$ \\
AST (U/L) & 20 & $38 \pm 11$ & $2-40$ & $9(45)$ \\
ALT (U/L) & 20 & $24 \pm 12$ & $0-35$ & $2(10)$ \\
GGTP (U/L) & 19 & $46 \pm 30$ & $5-40$ & $9(47)$ \\
Bilirubin (mmol/L) & 20 & $0.8 \pm 0.7$ & $0.3-1.2$ & $1(5)$
\end{tabular}

$\overline{S D}$, Standard deviation; $P T$, prothrombin time; PPT, partial thromboplastin time; $A S T$, aspartate aminotransferase; $A L T$, alanine aminotransferase; GGTP, gammaglutamyl transpepsidase.

procoagulant factors and inhibitors of coagulation were demonstrated in all children. Although there was no correlation between the significantly low levels of various factors with hemodynamic variables or liver function tests, the significant elevation in factor VIII, and its correlation with an increased SVC pressure, could suggest a prothrombotic relationship in patients with Fontan physiology.

The cause of thromboembolic events after the Fontan procedure seems to be multifactorial, and no consistent predisposing risk factors have been identified. Several authors have previously described coagulation factor abnormalities involving both pro- and anticoagulant proteins as a cause of a hypercoagulable state in children who have previously undergone the Fontan operation, with specific emphasis on low levels of the naturally occurring inhibitors of coagulation, protein $\mathrm{C}$, protein $\mathrm{S}$ and, ATIII. ${ }^{7-10}$ These earlier studies suggested that an imbalance between procoagulant factors and inhibitors of coagulation favors thrombus formation and that one or more aspects of the Fontan physiology may be responsible for the imbalance. However, these studies did not examine a full complement of coagulation factors and did not employ age-matched control subjects, nor did they undertake detailed hemodynamic evaluation.

The use of age-matched controls is important because the hemostatic system matures over the first several years of life, ${ }^{13}$ leading to important differences in factor activity measurements in children compared with adults. In general, values of most pro- and anticoagulant factors are lowest in neonates and infants and increase toward adult values at 
varying rates, some not reaching adult levels until relatively late in the teenage years. Despite the observed differences there is no evidence that infants are at greater risk for hemorrhagic or thrombotic problems compared with adults, suggesting that the neonatal and infant system is in "functional balance" at lower concentrations of most factors. Whether the overall coagulation factor abnormalities (compared to age-matched controls) we measured in our patients are due to delayed maturation in the Fontan patient is speculative.

Hepatic synthetic dysfunction (eg, reduced albumin) or evidence of hepatocellular dysfunction (eg, bilirubin, transaminase) did not appear to be associated with coagulation abnormalities in our patients, an important observation given the prominent role of the liver in the synthesis of procoagulants and inhibitors of coagulation and the potential for altered liver blood flow and oxygen delivery in Fontan patients. We were unable to relate specific hemodynamic variables, including an elevated SVCp or poor ventricular function, with altered hepatic synthetic function.

We have previously reported that abnormalities in coagulation factor levels may occur earlier in the staged palliation of complex single ventricle disease. ${ }^{11,12}$ The changes after the Fontan procedure found in our present study are qualitatively and quantitatively similar to those found in patients prior to the Fontan operation, with the important exception of factor VIII. In contrast to the two prior stages of single ventricle repair (pre-Glenn and pre-Fontan) where factor VIII concentrations were lower than age-matched controls, it appears that Fontan physiology is associated with a specific tendency to increase factor VIII; in 6 patients the level was significantly above the normal range. A similar finding was recently reported by Rauch and colleagues in 20 patients 4 to 63 months following the total cavopulmonary connection. ${ }^{14}$

The importance of an elevated factor VIII level as an independent risk factor for venous thrombosis in patients without cardiac defects has been reported. ${ }^{15-19}$ Factor VIII levels $>150 \%$ are associated with a five- to sixfold increased risk for venous thrombosis when compared with levels below $100 \%{ }^{20}$ The overall prevalence of an elevated factor VIII appears to be relatively high, having been reported in one study to be elevated in $25 \%$ of patients with a first episode of venous thrombosis and $11 \%$ of the healthy population. ${ }^{18}$ In a prospective study by Kyrle and colleagues of 360 adult patients, the risk of recurrence of thromboembolism was almost 7 times as great among patients with factor VIII levels above the 90th percentile $(>234 \%),{ }^{15}$ and it was speculated that prolonged anticoagulation therapy may be indicated in this subset of patients.

The cause of increased factor VIII levels in patients with Fontan circulation is unknown. The apparent conversion from a state of relatively low factor VIII earlier in the course of single ventricle repair suggests an acquired disturbance related directly to Fontan physiology. This is supported in our study by the significant relationship demonstrated between factor VIII activity and the higher SVC pressure. Factor VIII is expressed by multiple tissues. A recent report by Hollestelle and colleagues ${ }^{21}$ showed that the liver and kidney especially express high levels of factor VIII mRNA compared with the level of expression of other hemostatic proteins in these organs. Further analysis indicated that most of the VIII mRNA expression was detected in the endothelial lining of the liver sinusoids; a significantly lower signal was detected in hepatocytes. Although speculative, it is possible that the Fontan circulation with elevated central and hepatic venous pressure may contribute to an increase in factor VIII production from liver sinusoidal endothelium. This could also explain the lower factor VIII levels detected in patients with bidirectional Glenn physiology who have lower atrial and hepatic venous pressure.

In view of the potential risk of thromboembolic events in post-Fontan patients and evidence that high levels of factor VIII may be an independent risk factor for both early and late thromboembolic events, quantifying factor VIII may identify a subset of Fontan patients who would benefit from anticoagulant treatment. Further work is clearly necessary to determine whether there may be a critical factor VIII plasma concentration associated with thromboembolic risk in these patients and also whether, analogous to adult venous thrombosis events, there is an interaction with low levels of native circulating inhibitors of coagulation (eg, proteins $\mathrm{C}$ and $\mathrm{S}$ and ATIII) or prothrombotic genetic mutations.

\section{References}

1. Stamm C, Friehs I, Mayer JE, Zurakowski D, et al. Long-term results of the lateral tunnel Fontan operation. J Thorac Cardiovasc Surg. 2001;121:28-41

2. Cromme-Dijkhuis AH, Hess J, Hahlen K, et al. Specific sequelae after Fontan operation at mid- and long-term follow-up. Arrhythmia, liver dysfunction, and coagulation disorders. J Thorac Cardiovasc Surg. 1993;106:1126-32.

3. Rosenthal DN, Friedman AH, Kleinman CS, Kopf GS, Rosenfeld LE, Hellenbrand WE. Thromboembolic complications after Fontan operations. Circulation. 1995;92(Suppl II):287-93.

4. Jahangiri M, Ross DB, Redington AN, Lincoln C, Shinebourne EA. Thromboembolism after the Fontan procedure and its modifications. Ann Thorac Surg. 1994;58:1409-14.

5. Balling G, Vogt M, Kaemmerer H, Eicken A, Meisner H, Hess J. Intracardiac thrombus formation after the Fontan operation. J Thorac Cardiovasc Surg. 2000;119:745-52.

6. Monagle P, Cochrane A, McCrindle B, Benson L, Williams W, Andrew M. Editorial: thromboembolic complications after the Fontan procedures - the role of prophylactic anticoagulation. J Thorac Cardiovasc Surg. 1998;115:493-8.

7. Jahangiri M, Shore D, Kakkar V, Lincoln C, Shinebourne E. Coagulation factor abnormalities after the Fontan procedure and its modifications. J Thorac Cardiovasc Surg. 1997;113:989-93.

8. Cromme-Dijkhuis AH, Henkens CM, Bijleveld CM, Hillege HL, Bom VJ, van der Meer J. Coagulation factor abnormalities as possible thrombotic risk factors after Fontan operations. Lancet. 1990;336: 1087-90.

9. van Nieuwenhuizen RC, Peters M, Lubbers LJ, Trip MD, Tijssen JG, Mulder BJ. Abnormalities in liver function and coagulation profile following the Fontan procedure. Heart. 1999;82:40-6. 
10. Jahangiri M, Kreutzer J, Zurakowski D, Bacha E, Jonas RA. Evaluation of hemostatic and coagulation factor abnormalities in patients undergoing the Fontan operation. J Thorac Cardiovasc Surg. 2000; 120:778-82.

11. Odegard KC, McGowan FX, DiNardo JA, et al. Coagulation factor abnormalities in patients with single ventricle physiology precede the Fontan operation. J Thorac Cardiovasc Surg. 2002;123:459-65.

12. Odegard KC, McGowan, FX, Zurakowski D, et al. Coagulation factor abnormalities in patients with single ventricle physiology immediately prior to the Fontan procedure. Ann Thorac Surg. 2002;73:1770-7.

13. Andrew M, Vegh P, Johnston M, Bowker J, Ofosu F, Mitchell L. Maturation of the hemostatic system during childhood. Blood. 1992; 80:1998-2005.

14. Rauch R, Ries M, Hofbeck M, Buheitel G, Singer H, Klinge J. Hemostatic changes following the modified Fontan operation (total cavopulmonary connection). Thromb Haemost. 2000;83:678-82.

15. Kyrle PA, Minar E, Hirschl M, et al. High Plasma levels of factor VIII and the risk of recurrent venous thromboembolism. $N$ Engl J Med. 2000;343:457-62.
16. O’Donnell J, Tuddenham EG, Manning R, Kemball-Cook G, Johnson D, Laffan M. High prevalence of elevated factor VIII levels in patients referred for thrombophilia screening: role of increased synthesis and relationship to the acute phase reaction. Thromb Haemost. 1997;77: 825-8.

17. Kamphuisen PW, Eikenboom JC, Vos HL, et al. Increased levels of factor VIII and fibrinogen in patients with venous thrombosis are not caused by acute phase reaction. Thromb Haemost. 1999;81:680-3.

18. Kraaijenhagen RA, in't Anker PS, Koopman MM, et al. High plasma concentration of factor VIIIc is a major risk factor for venous thrombembolism. Thromb Haemost. 2000;83:5-9.

19. Kamphuisen PW, Eikenboom PW, Bertina RM. Elevated factor VIII levels and the risk of thrombosis. Arterioscler Thromb Vasc Biol. 2001;21:731-8.

20. Koster T, Blann AD, Briet E, Vandenbroucke JP, Rosendaal FR. Role of clotting factor VIII in effect of von Willebrand factor on occurrence of deep-vein thrombosis. Lancet. 1995;345:152-5.

21. Hollestelle MJ, Thinnes T, Crain K, et al. Tissue distribution of factor VIII gene expression in vivo-a closer look. Thromb Haemost. 2001; $86: 855-61$

\section{UN THE MOVE?}

Don't miss a single issue of the journal! To ensure prompt service when you change your address, please photocopy and complete the form below.

Please send your change of address notification at least six weeks before your move to ensure continued service. We regret we cannot guarantee replacement of issues missed due to late notification.

\section{JOURNAL TITLE:}

Fill in the title of the journal here.

OLD ADDRESS:

Affix the address label from a recent issue of the journal here.
NEW ADDRESS:

Clearly print your new address here.

Name

Address

City/State/ZIP
COPY AND MAIL THIS FORM TO:

Mosby

Subscription Customer Service

6277 Sea Harbor Dr

Orlando, FL 32887
OR FAX TO:

407-363-9661

N/M Mosby
OR PHONE:

800-654-2452

Outside the U.S., call

407-345-4000 